\title{
Autaptic Modulation of Electrical Activity in a Network of Neuron-Coupled Astrocyte
}

\author{
Shengli Guo, ${ }^{1}$ Jun Tang, ${ }^{2}$ Jun Ma, ${ }^{1,3}$ and Chunni Wang ${ }^{1}$ \\ ${ }^{1}$ Department of Physics, Lanzhou University of Technology, Lanzhou 730050, China \\ ${ }^{2}$ School of Physics, China University of Mining and Technology, Xuzhou 221116, China \\ ${ }^{3}$ NAAM-Research Group, Department of Mathematics, Faculty of Science, King Abdulaziz University, \\ P.O. Box 80203, Jeddah 21589, Saudi Arabia
}

Correspondence should be addressed to Jun Ma; hyperchaos@163.com

Received 10 April 2017; Accepted 9 May 2017; Published 5 June 2017

Academic Editor: Christos Volos

Copyright (C) 2017 Shengli Guo et al. This is an open access article distributed under the Creative Commons Attribution License, which permits unrestricted use, distribution, and reproduction in any medium, provided the original work is properly cited.

\begin{abstract}
Autapse connection is considered on a biological neuron coupled by astrocyte, and the effect of autapse driving-induced response in electrical activities is investigated. In this paper, a simple network is developed on the Hodgkin-Huxley $(\mathrm{HH})$ neuron coupled by astrocyte and the autapse effect is also considered. The modulation of autapse connected to HH neuron can change the membrane potential by applying time-delayed feedback along a close loop. It is found that the self-adaption of autapse driving can make the network of neuron-astrocyte generate different modes of electrical activities, and oscillating behavior of $\mathrm{Ca}^{2+}$ and $\mathrm{IP}_{3}$ setting is controlled. This new network model can give potential understanding about self-adaption of neuron to external forcing when the coupling of astrocyte and autapse is considered.
\end{abstract}

\section{Introduction}

It is ever believed that neuron is the most important basic unit of nerve system, and many experimental evidences have confirmed that gliocyte, particularly, astrocyte, can play an important role in changing the fluctuation of membrane potential of neuron by adjusting the concentration of $\mathrm{Ca}^{2+}$ via $\mathrm{IP}_{3}$ (inositol triphosphate) [1-3]. It is also found that astrocyte can format the inputting and activity of synapse $[4,5]$ and regulate the processing propagation of electrical signal in neurons [6-9]. Autapse [10-15] is often connected to some moderate neurons, and the feedback on membrane potential is realized by adding time-delayed forcing current on the membrane, where autapse is a specific synapse connected to the neuron via close loop. When autapse connection is triggered, the electrical activities and dynamical response will be changed [16-18]. Furthermore, autapse driving in the network can regulate the collective behaviors of network by generating continuous pulse or wave fronts, thus synchronization; pattern selection can be realized [19-22]. Readers can find brief survey for neurodynamics and dynamics in neuronal network in [23] and references therein. The author of this paper ever explained the formation mechanism of autapse connection to neuron; it is argued that formation of autapse can be helpful to enhance signal propagation along an auxiliary loop; thus autapse is developed [24]; then time delay and feedback gain are used to describe the properties of this loop. On the other hand, the electrical activities of neurons can be changed when neurons or neuronal tissue are exposed to electromagnetic radiation; for example, failure in heart induced by electromagnetic radiation [25] is discussed. In fact, complex electromagnetic induction is triggered in neurons during the exchange of ions current across membrane because the distribution of charged ions is changed. Therefore, Lv et al. suggested that magnetic flux [26, 27] can be used to detect the effect of electromagnetic induction and further for the effect of electromagnetic radiation [28]. Furthermore, Xu et al. argued [29] that autaptic driving can be helpful for neuron to suppress the electromagnetic radiation. Therefore, it is important to further discuss the network connection of neurons that the self-adaption of autapse connection can be understood. 
In fact, most of the previous works about neurodynamics have discussed the neuron-coupled astrocyte model [3034] and some results could be helpful to understand the occurrence of seizure-like behavior [35]. Based on the wellknown neuron models, networks with different topological connections have been set to investigate the synchronization stability [36, 37], pattern selection, and mode transition in collective behaviors [38-45]. Indeed, reliable and biophysical neuron model is critical and important for further investigation on neurodynamics and potential mechanism of some neuronal diseases [46]. Therefore, it is interesting to set a more reliable neuron model that the effect of autapse driving and astrocyte on electrical activities can be explored. In this paper, we propose an improved neuron model coupled by astrocyte and autapse connection is also considered. The modulation of autapse driving on electrical activities in astrocyte-coupled neuron will be discussed, and the exchange of signal between astrocyte and neurons can be detected and understood.

\section{Model Setting and Description}

For simplicity but biophysical meaning, Hodgkin-Huxley neuron model will be driven by autaptic current, and additive modulation from astrocyte will be considered; it reads as follows:

$$
\begin{aligned}
C_{m} \frac{d V}{d t}= & g_{k} n^{4}\left(V_{k}-V\right)+g_{\mathrm{Na}} m^{3} h\left(V_{\mathrm{Na}}-V\right) \\
& +g_{L}\left(V_{L}-V\right)+I_{\text {ext }}+I_{\text {astro }}+I_{\text {aut }} \\
\frac{d m}{d t}= & \alpha_{m}(V)(1-m)-\beta_{m}(V) m \\
\frac{d h}{d t}= & \alpha_{h}(V)(1-h)-\beta_{h}(V) h \\
\frac{d n}{d t}= & \alpha_{n}(V)(1-n)-\beta_{n}(V) n,
\end{aligned}
$$

where the coefficients [7] for (1) are defined by

$$
\begin{aligned}
& \alpha_{m}=0.1 \frac{25-V}{\exp [(25-V) / 10]-1} ; \\
& \beta_{m}=4 \exp \left[\frac{-V}{18}\right] ; \\
& \alpha_{h}=0.07 \exp \left[\frac{-V}{20}\right] ; \\
& \beta_{h}=\frac{1}{\exp [(30-V) / 10]+1} ; \\
& \alpha_{n}=0.01 \frac{10-V}{\exp [(10-V) / 10]-1} ; \\
& \beta_{n}=0.125 \exp \left[\frac{-V}{80}\right],
\end{aligned}
$$

where $V$ is the membrane potential, $m, n, h$ are the gate variable, and $I_{\text {ext }}$ denotes an external forcing current, respectively.
$I_{\text {aut }}$ represents the autaptic current from autapse connection to the neuron, and in case of electric autapse driving, the autaptic current is calculated as follows:

$$
I_{\mathrm{aut}}=g_{e}(V(t-\tau)-V(t))
$$

where $g_{e}$ and $\tau$ are the feedback gain and time delay, respectively. Positive feedback is triggered to excite and enhance the oscillating behavior by setting negative values for $g_{e}$, while positive value for $g_{e}$ can generate negative feedback to suppress the excitability and bursting behaviors in neuron. $I_{\text {astro }}$ defines the additive forcing current generated by astrocyte which changes the concentration of calcium and inositol triphosphate $\left(\mathrm{IP}_{3}\right)$ via adjusting the neurotransmitter such as ATP and glutamic acid. The modulation for concentration of $\mathrm{IP}_{3}$ can be approached by

$$
\begin{aligned}
\frac{d\left[\mathrm{IP}_{3}\right]}{d t}= & \frac{1}{\tau_{\mathrm{IP}_{3}}}\left(\left[\mathrm{IP}_{3}\right]^{*}-\left[\mathrm{IP}_{3}\right]\right) \\
& +r_{\mathrm{IP}_{3}} \Theta(V-50.0 \mathrm{mV}),
\end{aligned}
$$

where $\left[\mathrm{IP}_{3}\right]^{*}$ is the concentration of $\mathrm{IP}_{3}$ under equilibrium state and the parameter $r_{\mathrm{IP}_{3}}$ represents the response efficiency of astrocyte to action potential, also called production ratio for $\mathrm{IP}_{3}$. That is, the larger $r_{\mathrm{IP}_{3}}$, the larger density of distribution of $\mathrm{mGluR}$ on the membrane. $\Theta(*)$ is Heaviside function; as a result, the astrocyte can change the electrical activities of neuron and generate $\mathrm{IP}_{3}$ when the action potential or membrane potential is beyond $50 \mathrm{mV}$. The fluctuation of $\mathrm{IP}_{3}$ makes the receptor of $\mathrm{IP}_{3}$ trigger release of calcium ion; thus concentration of calcium ion $\left[\mathrm{Ca}^{2+}\right]$ began to oscillate. For simplicity, Li-Rinzel [47] model is used to describe the oscillating of $\left[\mathrm{Ca}^{2+}\right]$; it is often calculated by

$$
\begin{aligned}
\frac{d\left[\mathrm{Ca}^{2+}\right]}{d t} & =-J_{\text {Channel }}(q)-J_{\text {Pump }}-J_{\text {Leak }} \\
\frac{d q}{d t} & =\alpha_{q}(1-q)-\beta_{q} q,
\end{aligned}
$$

where $J_{\text {Channel }}(q)$ denote the calcium ion flux emitted from endoplasmic reticulum to cytoplasm via channels of $\mathrm{IP}_{3}$ receptor and thus the concentration of calcium ion is increased. $J_{\text {Pump }}$ is ATP-independent pump flux that calcium ion is pumped into calcium store. $J_{\text {Leak }}$ is leakage current from endoplasmic reticulum to cytoplasm. Order parameter $q$ is 
the gate variable that calculates the open probability of ion channels. The calcium flux [34] is described by

$$
\begin{aligned}
J_{\text {Channel }}(q) & =c_{1} v_{1} m_{\infty}^{3} n_{\infty}^{3} q^{3}\left(\left[\mathrm{Ca}^{2+}\right]-\left[\mathrm{Ca}^{2+}\right]_{\mathrm{ER}}\right) \\
J_{\text {Pump }} & =\frac{v_{3}\left[\mathrm{Ca}^{2+}\right]^{2}}{k_{3}^{2}+\left[\mathrm{Ca}^{2+}\right]^{2}} \\
J_{\text {Leak }} & =c_{1} v_{2}\left(\left[\mathrm{Ca}^{2+}\right]-\left[\mathrm{Ca}^{2+}\right]_{\mathrm{ER}}\right) \\
m_{\infty} & =\frac{\left[\mathrm{IP}_{3}\right]}{\left[\mathrm{IP}_{3}\right]+d_{1}} \\
n_{\infty} & =\frac{\left[\mathrm{Ca}^{2+}\right]}{\left[\mathrm{Ca}^{2+}\right]+d_{5}} \\
\alpha_{q} & =a_{2} d_{2} \frac{\left[\mathrm{IP}_{3}\right]+d_{1}}{\left[\mathrm{IP}_{3}\right]+d_{3}} \\
\beta_{q} & =a_{2}\left[\mathrm{Ca}^{2+}\right]
\end{aligned}
$$

And the forcing current from astrocyte is often approached by [30]

$$
\begin{aligned}
I_{\text {astro }} & =2.11 \Theta(\ln y) \ln y \\
y & =\left[\mathrm{Ca}^{2+}\right] / \mathrm{nM}-196.69 .
\end{aligned}
$$

As reported in [48-50], many chemical neurotransmitters are released to gaps of cells when the concentration of calcium ions is increased; for example, it is argued that the release of glutamic acid can trigger the release of calcium ions. However, blocking the transmission of glutamic acid between astrocytes seldom prevents the release of glutamic acid induced by increase of calcium ion concentration; it could account that glutamic acid comes from the interior of cells. It is believed that concentration increase in calcium ions is necessary setting to trigger the release of glutamic acid. When glutamic acid is released to gaps of cells, it is used as neurotransmitter to act on the ionophilic receptors (NMDA, AMPA), and depolarization of neuron occurs to trigger an action potential; as a result, signal propagation from synapse is regulated. It is found in [50] that slow introverted currents (SICs) via NMDA receptor can connect to thalamus neurons when pulse induced by $\mathrm{Ca}^{2+}$ oscillating in concentration was released and propagated to gaps between cells. For simplicity, the forcing current associated with astrocyte dependence on calcium concentration is approached in (7) described

\begin{tabular}{|c|c|}
\hline Parameter & Value and meaning \\
\hline$C_{m}$ & $1 \mu \mathrm{F} / \mathrm{cm}^{2}$ \\
\hline$g_{K}$ & $36.0 \mathrm{~ms} / \mathrm{cm}^{2}$ \\
\hline$g_{\mathrm{Na}}$ & $120.0 \mathrm{~ms} / \mathrm{cm}^{2}$ \\
\hline$g_{L}$ & $0.3 \mathrm{~ms} / \mathrm{cm}^{2}$ \\
\hline$V_{K}$ & $-12.0 \mathrm{mV}$ \\
\hline$V_{\mathrm{Na}}$ & $115.0 \mathrm{mV}$ \\
\hline$V_{L}$ & $10.6 \mathrm{mV}$ \\
\hline$\left[\mathrm{IP}_{3}\right]^{*}$ & $160.0 \mathrm{nmol} / \mathrm{L}$ \\
\hline $1 / \tau_{\mathrm{IP}_{3}}$ & $0.00014(\mathrm{~m} / \mathrm{s})$ \\
\hline$c_{0}$ & $2.0 \mu \mathrm{mol} / \mathrm{L}$ \\
\hline$c_{1}$ & 0.185 \\
\hline$v_{1}$ & $6 s^{-1}$ \\
\hline$v_{2}$ & $0.11 \mathrm{~s}^{-1}$ \\
\hline$v_{3}$ & $0.9 \mu \mathrm{mol} / \mathrm{L} \cdot \mathrm{s}$ \\
\hline$k_{3}$ & $0.1 \mu \mathrm{mol} / \mathrm{L}$ \\
\hline$d_{1}$ & $0.13 \mu \mathrm{mol} / \mathrm{L}$ \\
\hline$d_{2}$ & $1.049 \mu \mathrm{mol} / \mathrm{L}$ \\
\hline$d_{3}$ & $0.9434 \mu \mathrm{mol} / \mathrm{L}$ \\
\hline$d_{5}$ & $0.08234 \mu \mathrm{mol} / \mathrm{L}$ \\
\hline$a_{2}$ & $0.2 \mu \mathrm{mol} / \mathrm{L} \cdot \mathrm{s}$ \\
\hline$r_{\mathrm{IP}_{3}}$ & Response efficiency of astrocyte to action potential \\
\hline$g_{e}$ & Feedback gain in autapse \\
\hline$\tau$ & Time delay in autapse \\
\hline
\end{tabular}
as above. The physical unit in astrocyte for calcium ions is $\mu \mathrm{mol} / \mathrm{L}, p A$ is used for current, and the cell or neuron is described as a sphere with a radius about $25 \mu \mathrm{m}$, and the density of current of $I_{\text {astro }}$ is $\mu \mathrm{mol} / \mathrm{L} \cdot \mathrm{cm}^{2}$ to be consistent with the physical units in Hodgkin-Huxley neuron model. For detailed description, Table 1 gives the parameter setting and physical units.
TABLE 1: Parameter values setting.

\section{Numerical Results and Discussion}

The fourth-order Runge-Kutta algorithm is presented to find numerical solution of membrane potential with time step 0.001 . The parameter $r_{\mathrm{IP}_{3}}$ is set to change different values, and the electric autapse driving is considered. Then the dynamical response in action potential is investigated to explore the possible biological function of autapse connection in the astrocyte-coupled neuron network. At first, the autapse connection is switched off, the external forcing is imposed on neuron as $I_{\text {ext }}=10.0 \mu \mathrm{mol} / \mathrm{L} \cdot \mathrm{cm}^{2}$ at $t<40 \mathrm{~s}$, the calcium concentration of astrocyte and $\mathrm{IP}_{3}$ is calculated at $r_{\mathrm{IP}_{3}}=0.2$, and the results are plotted in Figure 1.

It is found that the membrane potential is decreased to quiescent state when the external forcing current is removed and the oscillating in $\mathrm{Ca}^{2+}$ is also stabilized, the mechanism is that astrocyte and neuron are coupled with weak intensity, insufficient $\mathrm{IP}_{3}$ is not effective to trigger continuous oscillation in calcium concentration, and the exchange of transmembrane current is suppressed. Now, the effect of autapse connection and driving is considered by activating the autapse connection; for example, time delay in electric autapse is set $\tau=2$, and positive feedback is investigated in Figure 2 by setting different feedback gains in the autapse.

It is found that the membrane potentials of neuron begin to fluctuate when the feedback gain in the electric autapse is increased beyond the threshold $\left(g_{e}=-0.48\right)$; furthermore, the $\mathrm{IP}_{3}$ and $\mathrm{Ca}^{2+}$ follow its oscillating behavior to modulate the membrane potential greatly. The potential 


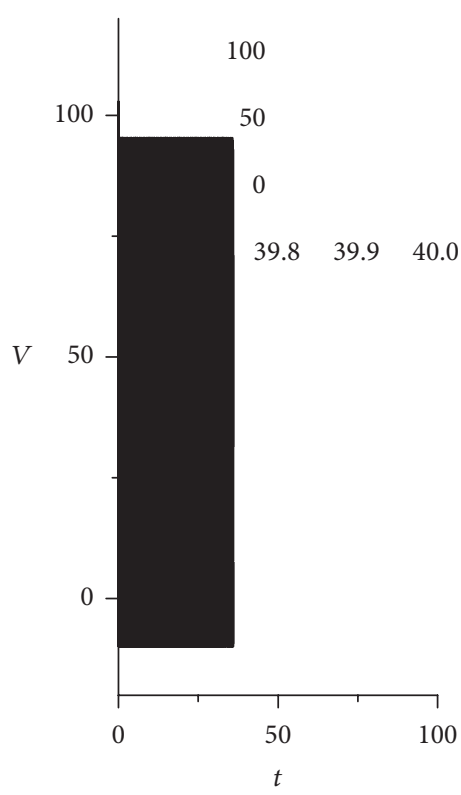

(a)

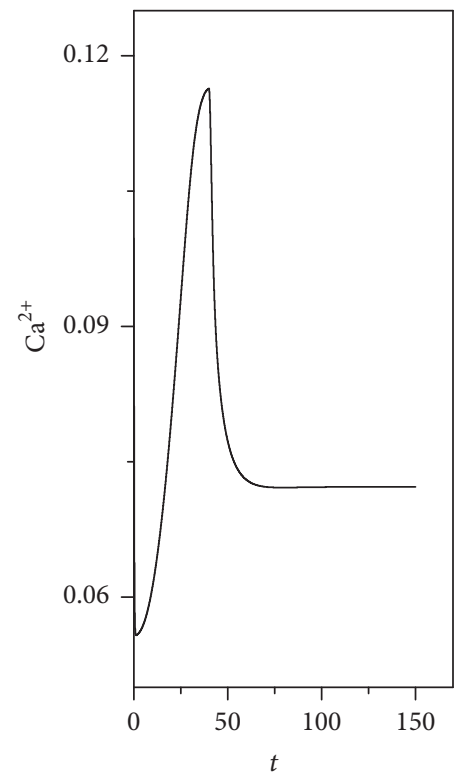

(b)

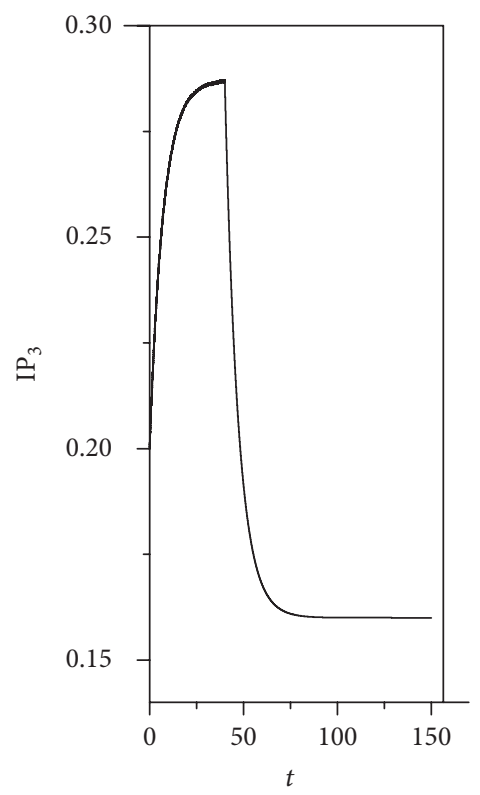

(c)

FIGURE 1: Sampled time series for membrane potential (a), calcium concentration in astrocyte $(\mathrm{b})$, and $\mathrm{IP}_{3}$ in astrocyte $(\mathrm{c}), r_{\mathrm{IP}}=0.2$, and the external forcing current regulates the neuronal activities by setting $I_{\text {ext }}=10.0 \mu \mathrm{mol} / \mathrm{L} \cdot \mathrm{cm}^{2}$ at $t<40 \mathrm{~s}$; the autaptic current is set as zero.

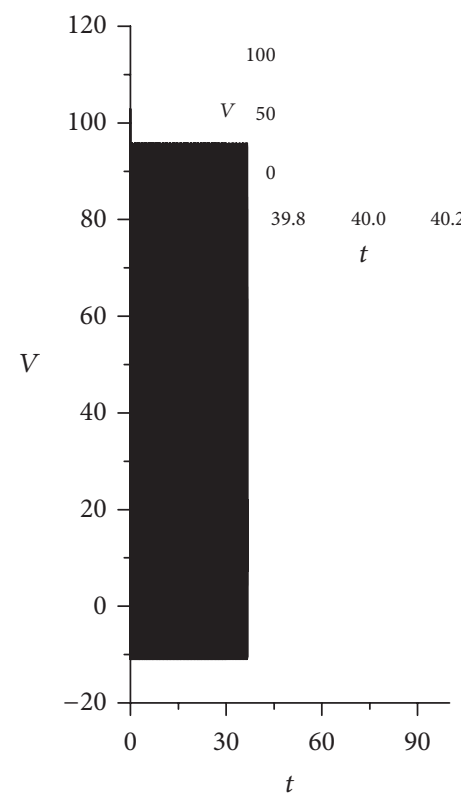

(a)

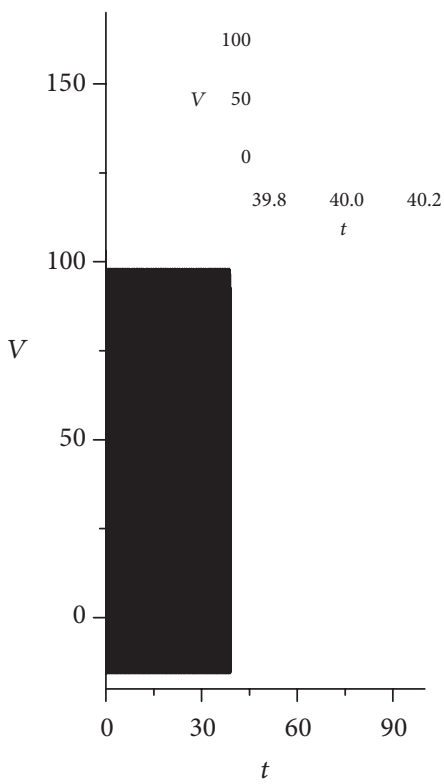

(b)

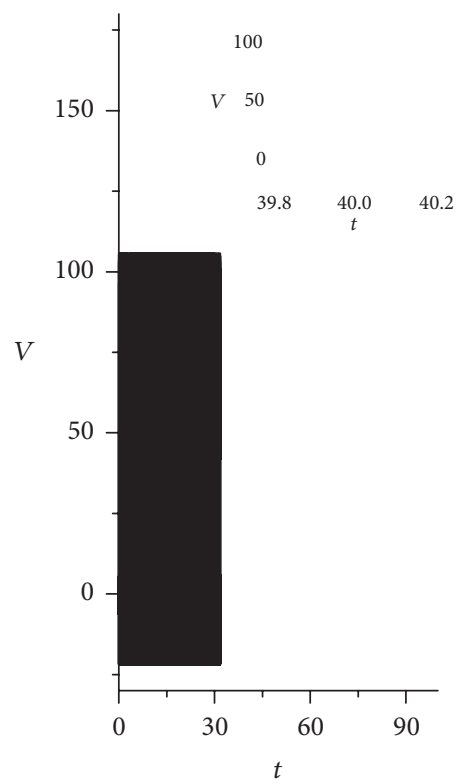

(c)

FIGURE 2: Sampled time series for membrane potential of neuron coupled by astrocypte when autapse driving is considered at $\tau=2$, for (a) $g_{e}=-0.1$, (b) $g_{e}=-0.5$, and (c) $g_{e}=-1.0$. The parameter is set as $r_{\mathrm{IP}_{3}}=0.2$, and inserted figures are enlarged ones. And the external forcing current regulates the neuron by $I_{\text {ext }}=10.0 \mu \mathrm{mol} / \mathrm{L} \cdot \mathrm{cm}^{2}$ at $t<40 \mathrm{~s}$.

mechanism is that positive feedback in autapse connection to neuron remembers the previous stimuli and continuous stimuli imposed on neuron can be effective to enhance the excitability of neuron, and then the neuron is excited. Furthermore, the oscillation of $\mathrm{Ca}^{2+}$ and changes of $\mathrm{IP}_{3}$ are calculated in Figure 3.
The results in Figure 3 confirmed that enough high concentration in $\mathrm{IP}_{3}$ is critical to trigger and enhance continuous oscillation of $\mathrm{Ca}^{2+}$ when electric autapse is set with strong feedback gain being applied. When the external forcing current is removed, the concentration of $\mathrm{IP}_{3}$ is decreased quickly because continuous release of neurotransmitter occurs in 


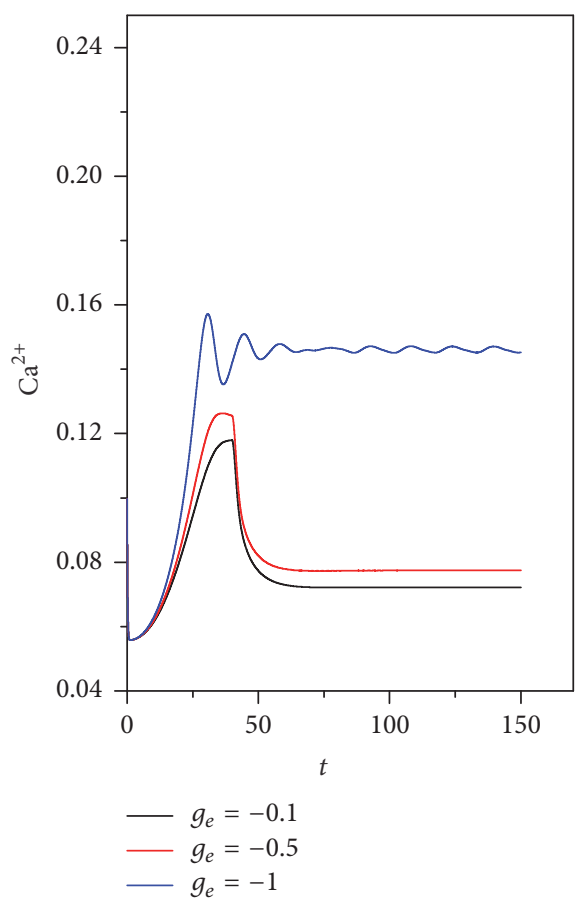

(a)

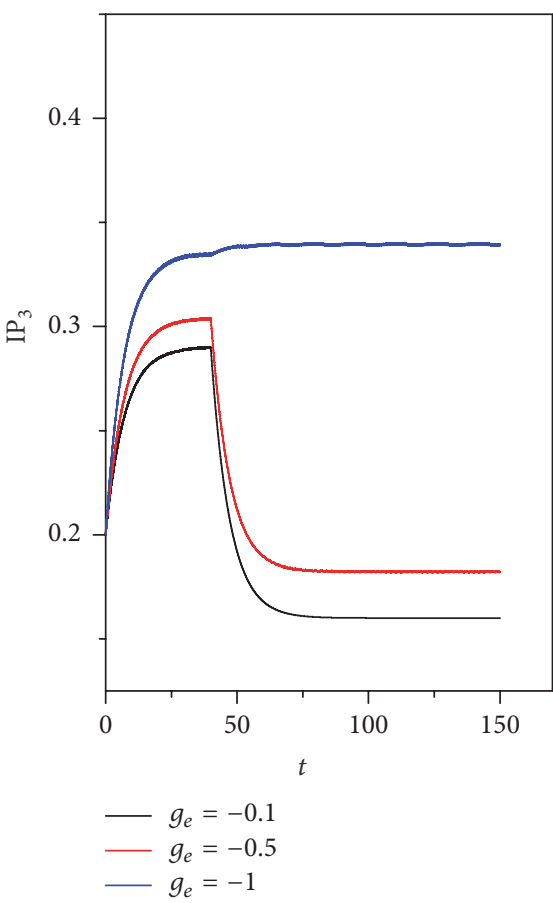

(b)

FIGURE 3: Fluctuation in $\mathrm{Ca}^{2+}$ concentration and $\mathrm{IP}_{3}$ concentration is calculated by applying different feedback gains $g_{e}$ in electric autapse with time delay $\tau=2$. The parameter is set as $r_{\mathrm{IP}_{3}}=0.2$. And the external forcing current regulates the neuron by setting $I_{\mathrm{ext}}=10.0 \mu \mathrm{mol} / \mathrm{L} \cdot \mathrm{cm}{ }^{2}$ at $t<40 \mathrm{~s}$.

presence of external stimuli beyond threshold. The interaction between neurotransmitter and metabolic receptor of astrocyte (mGluRs) can produce more $\mathrm{IP}_{3}$; as a result, $\mathrm{IP}_{3}$ independent $\mathrm{Ca}^{2+}$ can induce rapid release of $\mathrm{Ca}^{2+}$ from endoplasmic reticulum. On the other hand, positive feedback in electric autapse can also increase the $\mathrm{IP}_{3}$ concentration; thus the concentration of $\mathrm{Ca}^{2+}$ can be enhanced; for example, $g_{e}=-1.0$. Furthermore, the case of negative feedback is considered by setting positive feedback gain in the electric autapse; the results are plotted in Figure 4.

It is found that the fluctuation of membrane potential can be suppressed, and the neuronal activities are changed to become quiescent state when autapse driving imposed negative feedback on the neuron. Furthermore, the $\mathrm{Ca}^{2+}$ and $\mathrm{IP}_{3}$ concentration are calculated in Figure 5.

That is, negative feedback in electric autapse can suppress the fluctuation of membrane potential and then the ion current of $\mathrm{Ca}^{2+}$; as a result, the concentration of $\mathrm{IP}_{3}$ is also decreased completely. Indeed, neuron can be induced to trigger hyperexcitability when neuron is coupled by astrocyte with stronger intensity (e.g., larger value setting for $r_{\mathrm{IP}_{3}}$ ). In case of smaller $r_{\mathrm{IP}_{3}}$, the positive feedback in electric autapse becomes dominant and very important to enhance the excitability of neuron and also the oscillation in $\mathrm{Ca}^{2+}$ concentration. Furthermore, larger $r_{\mathrm{IP}_{3}}(=0.8)$ is used to investigate the same problem by applying external forcing current $I_{\text {ext }}=10.0 \mu \mathrm{mol} / \mathrm{L} \cdot \mathrm{cm}^{2}$ with a transient period $t=40 \mathrm{~s}$ from beginning, and the results are plotted in Figure 6.
It is confirmed that oscillating behavior of $\mathrm{Ca}^{2+}$ concentration and increase in $\mathrm{IP}_{3}$ concentration can also be detected by setting larger coupling intensity between neuron and astrocyte (larger $r_{\mathrm{IP}_{3}}$ ) when autapse connection is removed or the autaptic current is set as zero. In case of large value setting for $r_{\mathrm{IP}_{3}}, \mathrm{Ca}^{2+}$ and $\mathrm{IP}_{3}$ show slight oscillation in concentration when external forcing current is removed. It is interesting to detect the inhibition effect on $\mathrm{Ca}^{2+}$ and $\mathrm{IP}_{3}$ oscillation in concentration by applying negative feedback in electric autapse, and the results are calculated in Figures 7 and 8 .

It is found in Figure 7 that the spiking behavior of neuron can be suppressed by electric autapse with negative feedback; even the coupling between neuron and astrocyte is enhanced by setting larger $r_{\mathrm{IP}_{3}}$. Furthermore, the changes of $\mathrm{Ca}^{2+}$ and $\mathrm{IP}_{3}$ are calculated in Figure 8.

It is consistent with the previous prediction that $\mathrm{Ca}^{2+}$ keeps oscillating and $\mathrm{IP}_{3}$ holds large concentration when autaptic modulation on membrane potential under negative feedback is weak because the astrocyte contributes more in regulating the membrane potential than electric autapse in this case. By further increasing in the negative feedback in electric autapse, the oscillating behavior in $\mathrm{Ca}^{2+}$ and $\mathrm{IP}_{3}$ will be suppressed completely due to the modulation of electric autapse. It is important to detect and discuss whether positive feedback in autapse can enhance the oscillating behaviors of $\mathrm{Ca}^{2+}$ and $\mathrm{IP}_{3}$ in case of larger value setting for $r_{\mathrm{IP}_{3}}$, and the results are shown in Figures 9 and 10. 


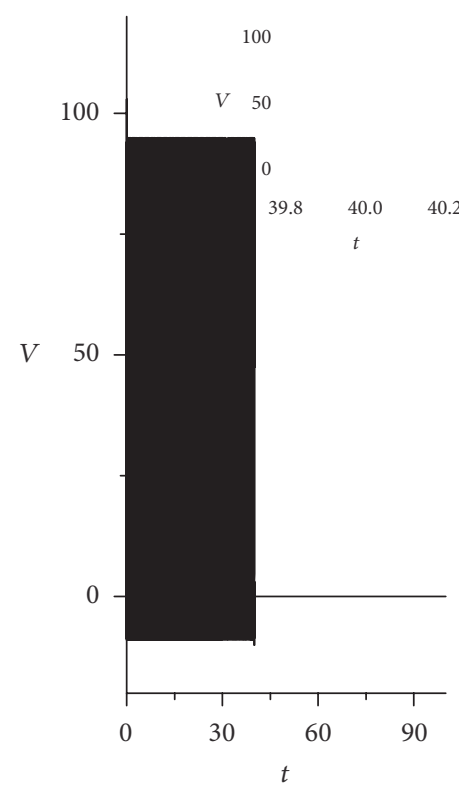

(a)

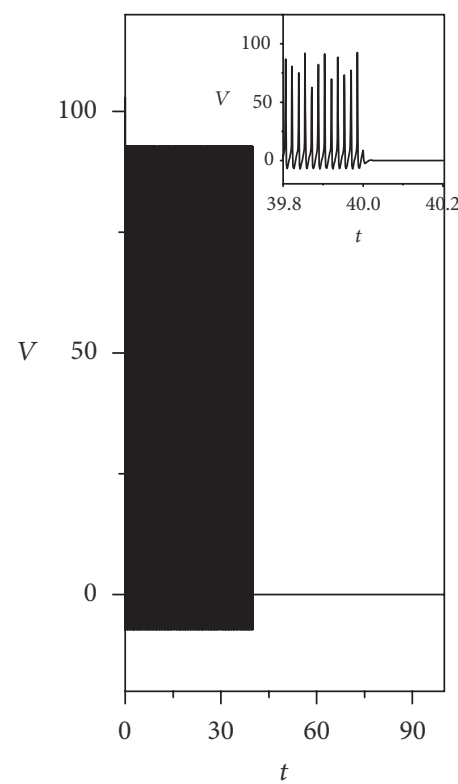

(b)

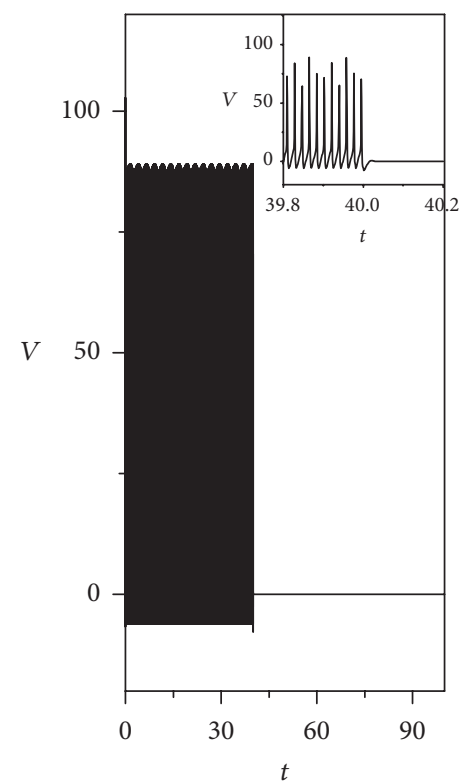

(c)

FIGURE 4: Sampled time series for membrane potential of neuron are calculated when neuron is coupled by astrocypte and autapse driving with negative feedback is considered at $\tau=2$, for (a) $g_{e}=0.1$, (b) $g_{e}=0.5$, and (c) $g_{e}=1.0$. The parameter is set as $r_{\mathrm{IP}_{3}}=0.2$, and inserted figures are enlarged ones. And the external forcing current regulates the neuron by $I_{\text {ext }}=10.0 \mu \mathrm{mol} / \mathrm{L} \cdot \mathrm{cm}^{2}$ at $t<40 \mathrm{~s}$. The inserted figures are enlarged to show the sampled time series for membrane potential from $t=39.8$ to $40.2 \mathrm{~ms}$.

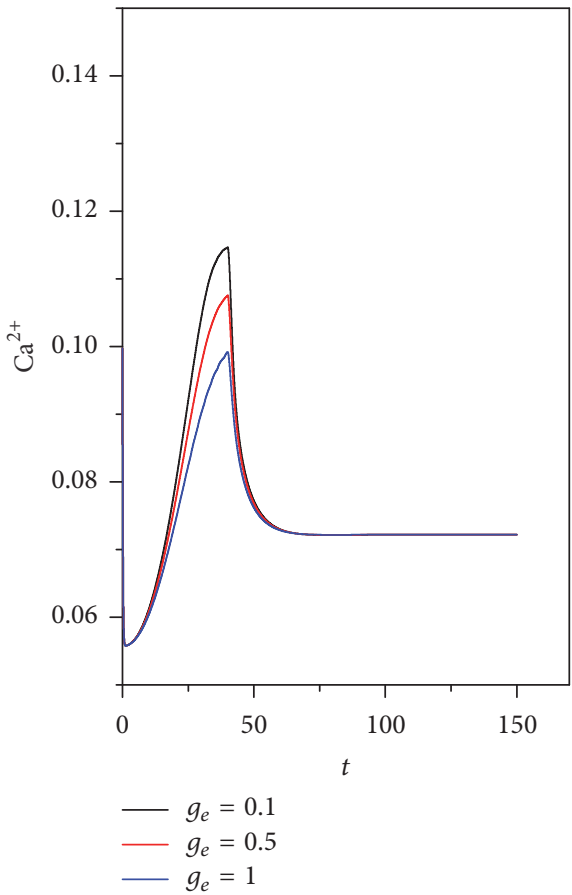

(a)

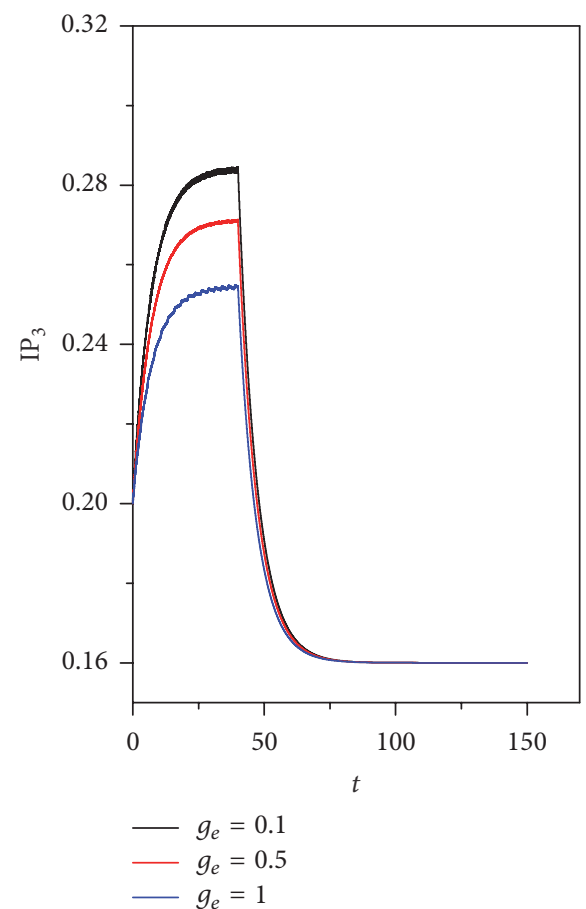

(b)

FIGURE 5: Fluctuation in $\mathrm{Ca}^{2+}$ concentration and $\mathrm{IP}_{3}$ concentration is calculated by applying different feedback gains $g_{e}$ in autapse with time delay $\tau=2$. The parameter is set as $r_{\mathrm{IP}_{3}}=0.2$. And the external forcing current regulates the neuron by setting $I_{\text {ext }}=10.0 \mu \mathrm{mol} / \mathrm{L} \cdot \mathrm{cm}^{2}$ at $t<40 \mathrm{~s}$. 


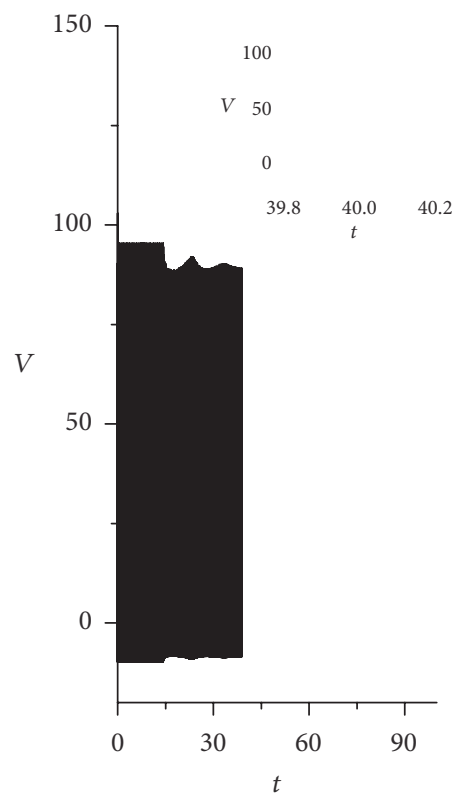

(a)

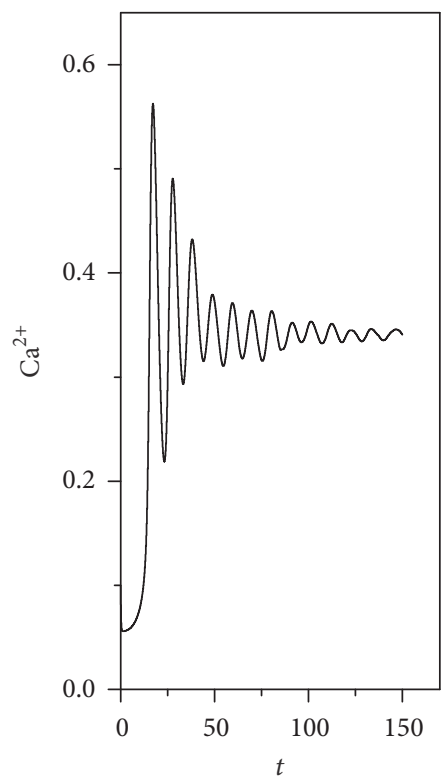

(b)

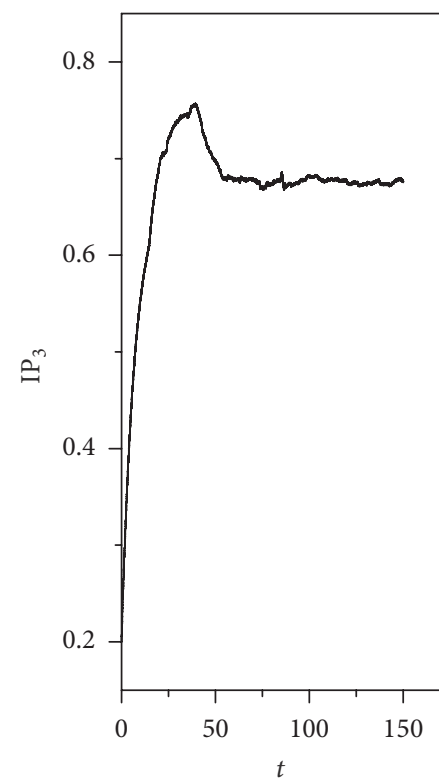

(c)

FIGURE 6: Sampled time series for membrane potential (a), calcium concentration in astrocyte (b), and $\mathrm{IP}_{3}$ in astrocyte $(\mathrm{c}), r_{\mathrm{IP}_{3}}=0.8$, and the external forcing current regulates the neuron by $I_{\text {ext }}=10.0 \mu \mathrm{mol} / \mathrm{L} \cdot \mathrm{cm}^{2}$ at $t<40 \mathrm{~s}$. The autaptic current is set as zero, and the inserted figure is enlarged to show the sampled time series for membrane potential from $t=39.8$ to $40.2 \mathrm{~ms}$.

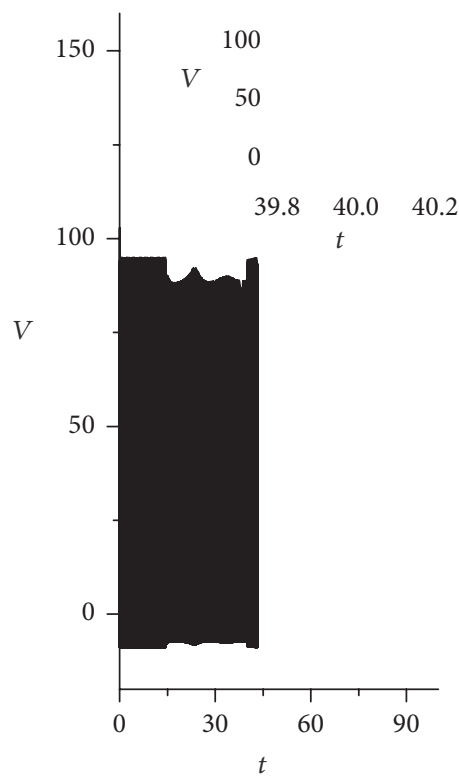

(a)

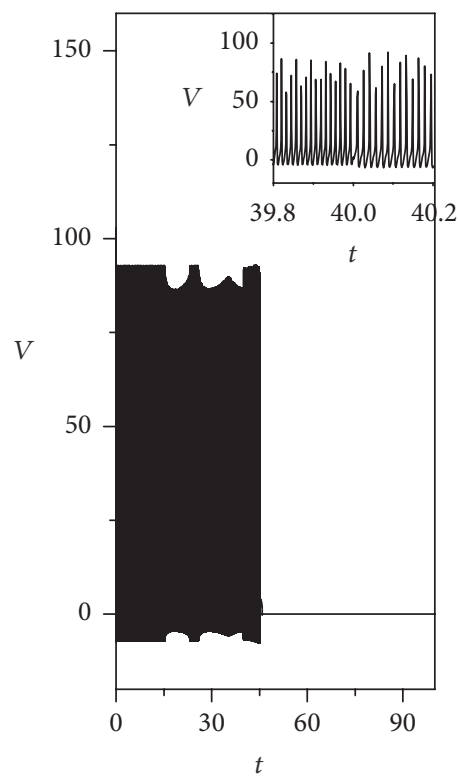

(b)

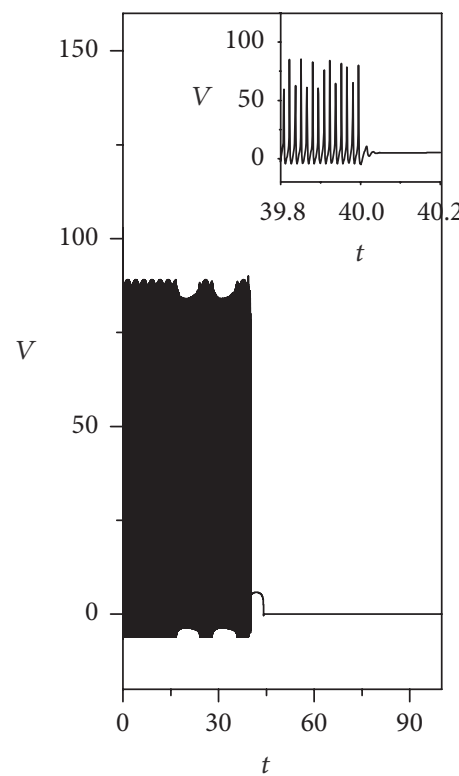

(c)

FIGURE 7: Sampled time series for membrane potential of neuron coupled by astrocypte when autapse driving is considered at $\tau=2$, for (a) $g_{e}=0.1$, (b) $g_{e}=0.5$, and (c) $g_{e}=1.0$. The parameter is set as $r_{\mathrm{IP}_{3}}=0.8$, and inserted figures are enlarged ones. And the external forcing current regulates the neuron by $I_{\text {ext }}=10.0 \mu \mathrm{mol} / \mathrm{L} \cdot \mathrm{cm}^{2}$ at $t<40 \mathrm{~s}$. The inserted figure is enlarged to show the sampled time series for membrane potential from $t=39.8$ to $40.2 \mathrm{~ms}$.

It is confirmed that positive feedback in electric autapse can further enhance the oscillating behavior of $\mathrm{Ca}^{2+}$ and $\mathrm{IP}_{3}$ and also the excitability of neuron when the coupling intensity between neuron and astrocyte is set as higher value. As it is known, the autaptic modulation in autapse also depends on the value setting for time delay, which is dependent on the close loop. As a result, different time delays in electric autapse are selected to check the response of electrical activities and the oscillating behaviors of $\mathrm{Ca}^{2+}$ and $\mathrm{IP}_{3}$, and the results are plotted in Figures 11 and 12. 


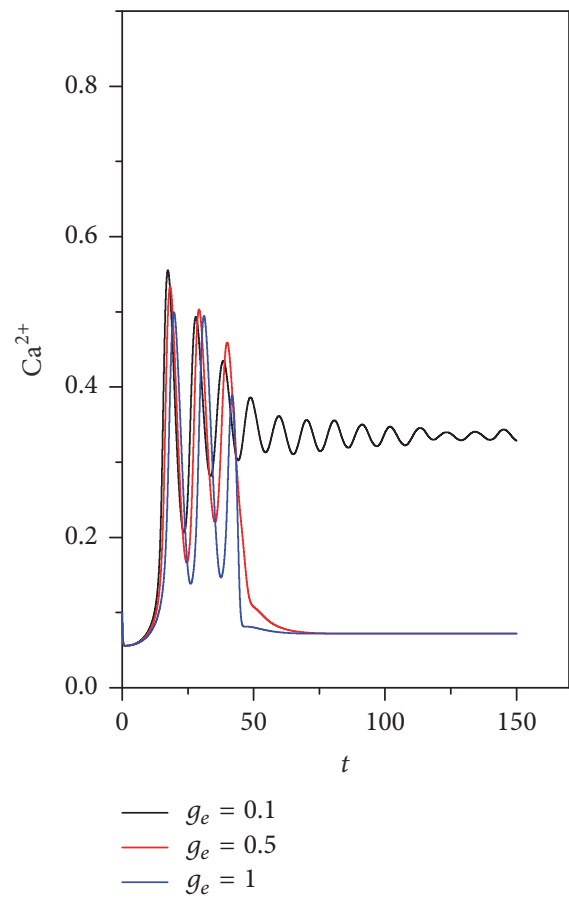

(a)

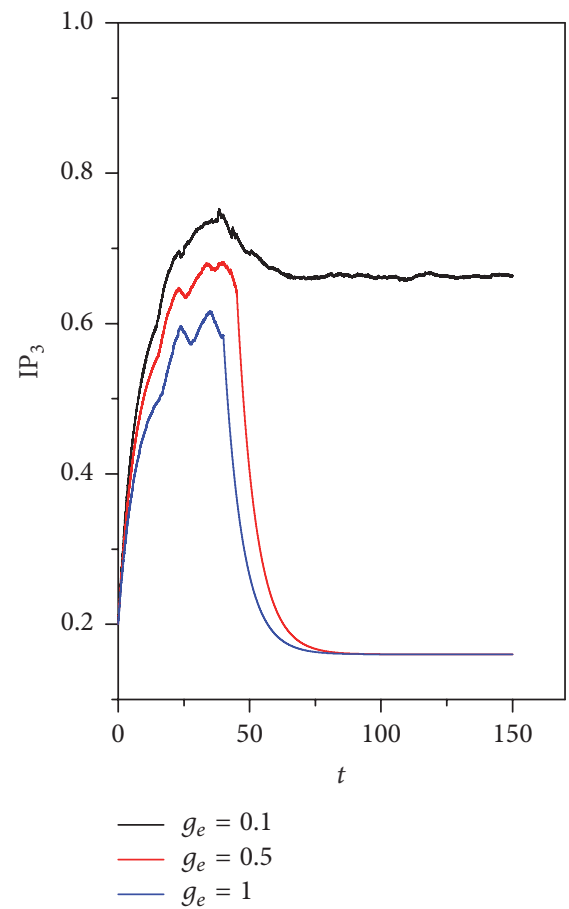

(b)

FIGURE 8: Fluctuation in $\mathrm{Ca}^{2+}$ concentration and $\mathrm{IP}_{3}$ concentration is calculated by applying different feedback gains $g_{e}$ in autapse with time delay $\tau=2$. The parameter is set as $r_{\mathrm{IP}_{3}}=0.8$. And the external forcing current regulates the neuron by $I_{\text {ext }}=10.0 \mu \mathrm{mol} / \mathrm{L} \cdot \mathrm{cm}{ }^{2}$ at $t<40 \mathrm{~s}$.

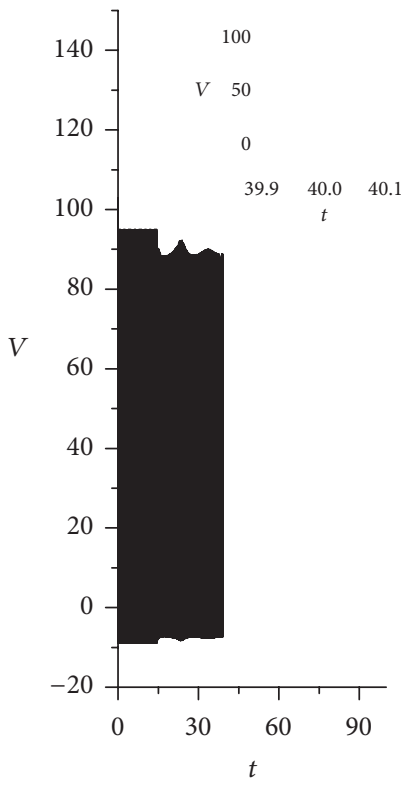

(a)

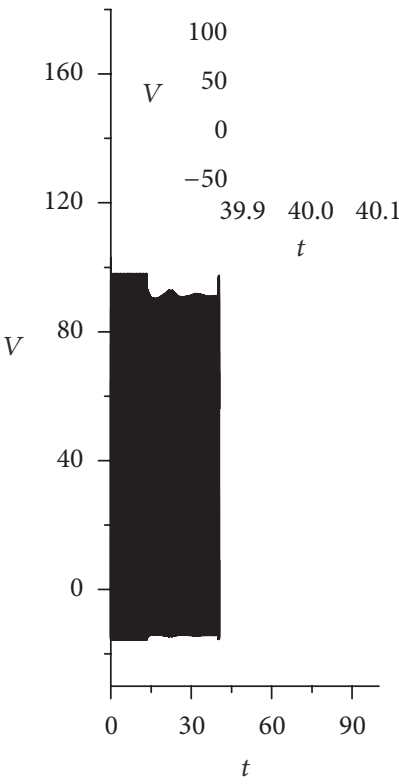

(b)

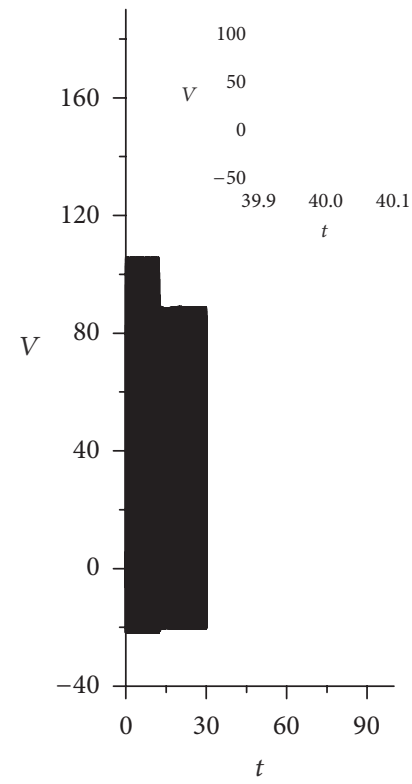

(c)

FIGURE 9: Sampled time series for membrane potential of neuron coupled by astrocypte when autapse driving is considered at $\tau=2$, for (a) $g_{e}=-0.1$, (b) $g_{e}=-0.5$, and (c) $g_{e}=-1.0$. The parameter is set as $r_{\mathrm{IP}_{3}}=0.8$, and inserted figures are enlarged ones. And the external forcing current regulates the neuron by $I_{\text {ext }}=10.0 \mu \mathrm{mol} / \mathrm{L} \cdot \mathrm{cm}^{2}$ at $t<40 \mathrm{~s}$. The inserted figure is enlarged to show the sampled time series for membrane potential from $t=39.8$ to $40.2 \mathrm{~ms}$. 


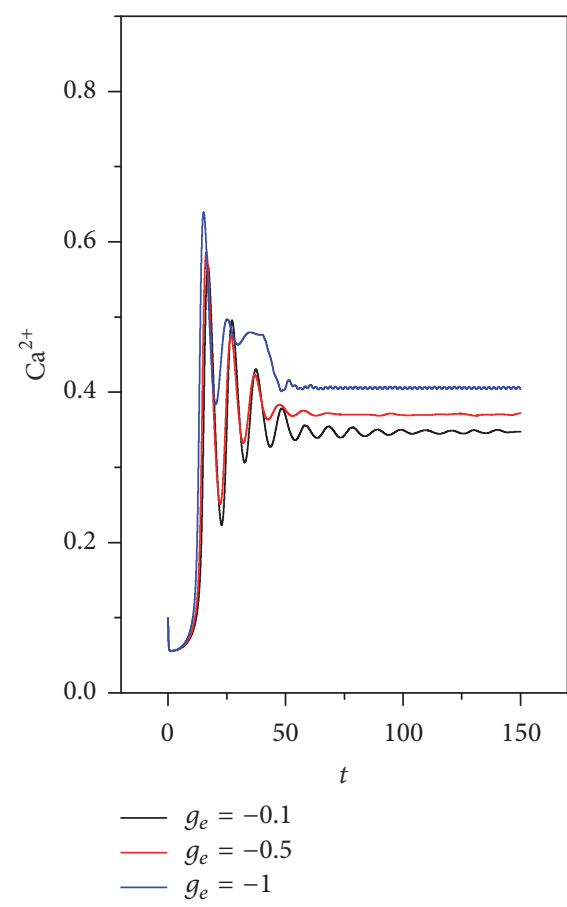

(a)

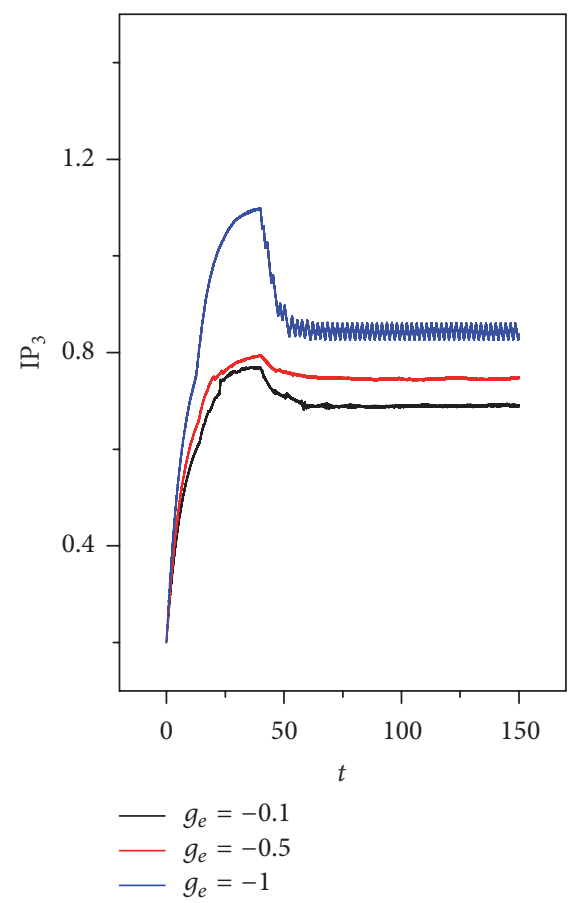

(b)

FIGURE 10: Fluctuation in $\mathrm{Ca}^{2+}$ concentration and $\mathrm{IP}_{3}$ concentration is calculated by applying different feedback gains $g_{e}$ in autapse with time delay $\tau=2$. The parameter is set as $r_{\mathrm{IP}_{3}}=0.8$. And the external forcing current regulates the neuron by setting $I_{\text {ext }}=10.0 \mu \mathrm{mol} / \mathrm{L} \cdot \mathrm{cm}^{2}$ at $t<40 \mathrm{~s}$.

It is found in Figure 11 that the spiking behavior is enhanced and began to show bursting behavior with increasing the time delay in electric autapse; even the coupling intensity between neuron and astrocyte is set as small value for $r_{\mathrm{IP}_{3}}=0.2$. Furthermore, the oscillating behavior of $\mathrm{Ca}^{2+}$ and changes of $\mathrm{IP}_{3}$ concentration are calculated in Figure 12.

It is confirmed in Figure 12 that the concentration of $\mathrm{IP}_{3}$ is decreased and the oscillating behavior in $\mathrm{Ca}^{2+}$ is also suppressed when the time delay in autapse is small. By further increasing the time delay in autapse, the memory effect makes neuron remember the action potential and oscillating behavior for $\mathrm{Ca}^{2+}$ though the intrinsic time delay in electric autapse is finite. Finally, the bifurcation analysis is carried out and the dependence of $\mathrm{Ca}^{2+}, \mathrm{IP}_{3}$, and ISI on parameters $r_{\mathrm{IP}_{3}}$ and $g_{e}$ is discussed in Figure 13, respectively.

It is confirmed that the consistent oscillating behaviors of $\mathrm{Ca}^{2+}$ and $\mathrm{IP}_{3}$ are dependent on the selection of $r_{\mathrm{IP}_{3}}$, and positive feedback in autapse is effective to enhance the oscillating behaviors and bursting firing in electrical activities. In fact, the $\mathrm{Ca}^{2+}$ oscillating in astrocyte is much complex when uncertain perturbation such as noise and time delay is considered [51-53]. Different disturbances on excitable media are often described by applying different types of noise (additive or multiplicative) on the media and possible statistical properties are discussed [54]. Mutual coupling between astrocyte and neuron driven by autapse can trigger complex stimuli for neuron, astrocyte by setting different external forcing currents, and time delay and feedback gain in autapse; as a result, the response of electrical activities becomes more complex. That is, both of autaptic modulation and astrocyte can cooperate and contribute the mode selection of electrical activities in neurons; thus the self-adaption of neurons can be enhanced.

In summary, autapse connection and driving, external forcing, and also the coupling between neuron and astrocyte all contribute the oscillating behavior for $\mathrm{Ca}^{2+}$ by increasing the $\mathrm{IP}_{3}$ concentration beyond the threshold to keep continuous oscillating in $\mathrm{Ca}^{2+}$ concentration. As a result, continuous action potential is triggered to propagate the electric signal between neurons. As mentioned in [24], formation and development of autapse can be associated with the selfadaption of neuron to external stimuli; particularly, it could be associated with injury in the neuron loop of circuit because only a few of neurons are found to be connected with autapse. Extensive evidences also confirmed that external setting of electric field can be effect to bridge the injured parts of axon; thus blocked signal can be propagated [55]. In this way, it could give guidance to further understand the formation mechanism of autapse; in the case of astrocyte modulation, the autaptic driving still plays an important role in regulating the signal exchange between neurons and astrocyte; these results could be helpful for further investigation on collective neuronal network composed of a large number of neurons coupled by astrocytes. 


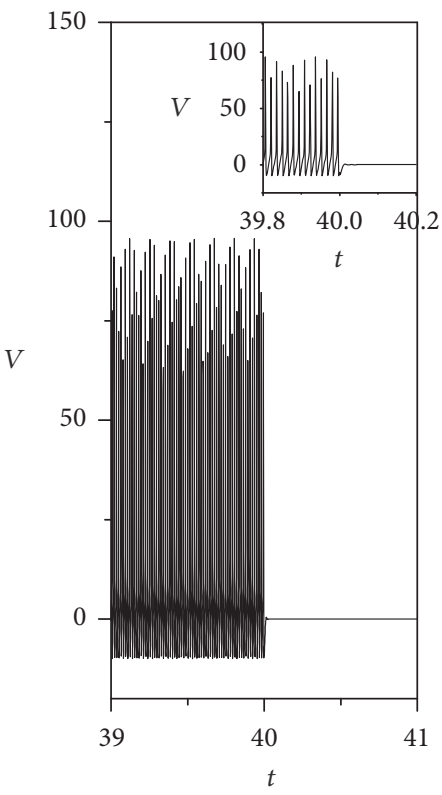

(a)

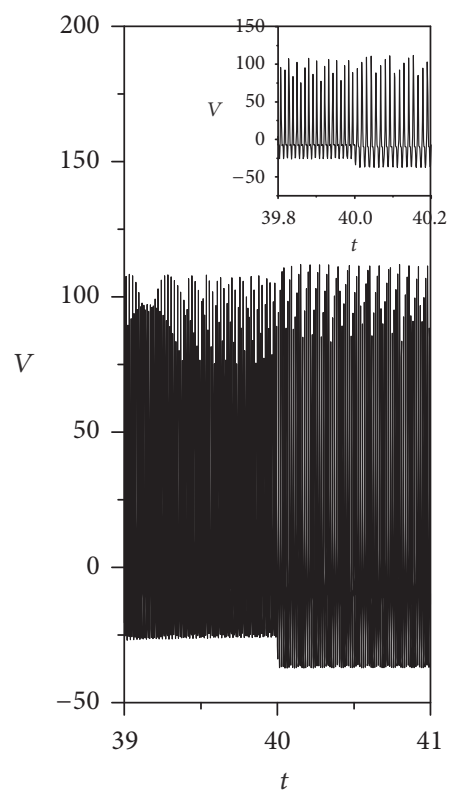

(b)

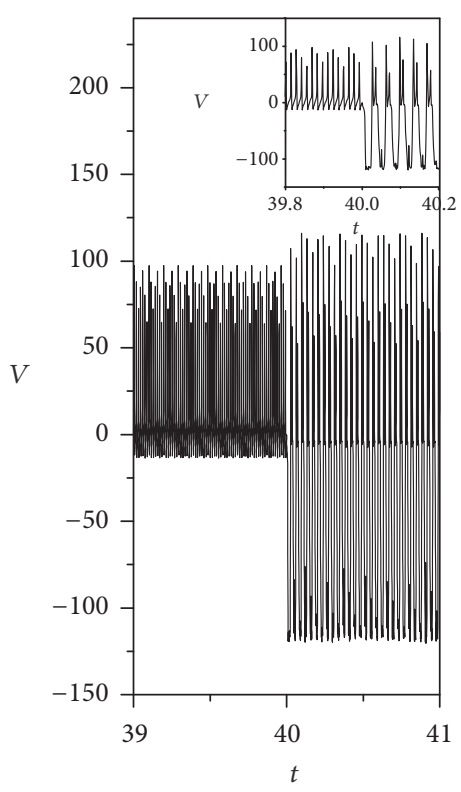

(c)

FIGURE 11: Sampled time series for membrane potential of neuron coupled by astrocypte when autapse driving is considered different time delays at $g_{e}=-0.5$, for (a) $\tau=0.1$, (b) $\tau=6$, and (c) $\tau=15$. The parameter is set as $r_{\mathrm{IP}_{3}}=0.2$, and inserted figures are enlarged ones. And the external forcing current regulates the neuron by setting $I_{\mathrm{ext}}=10.0 \mu \mathrm{mol} / \mathrm{L} \cdot \mathrm{cm}^{2}$ at $t<40 \mathrm{~s}$. The inserted figure is enlarged to show the sampled time series for membrane potential from $t=39.8$ to $40.2 \mathrm{~ms}$.

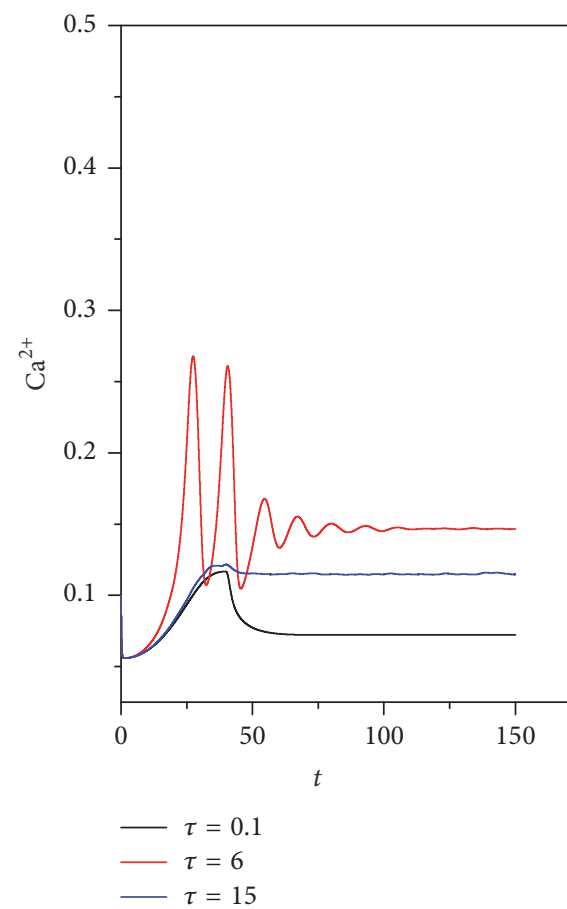

(a)

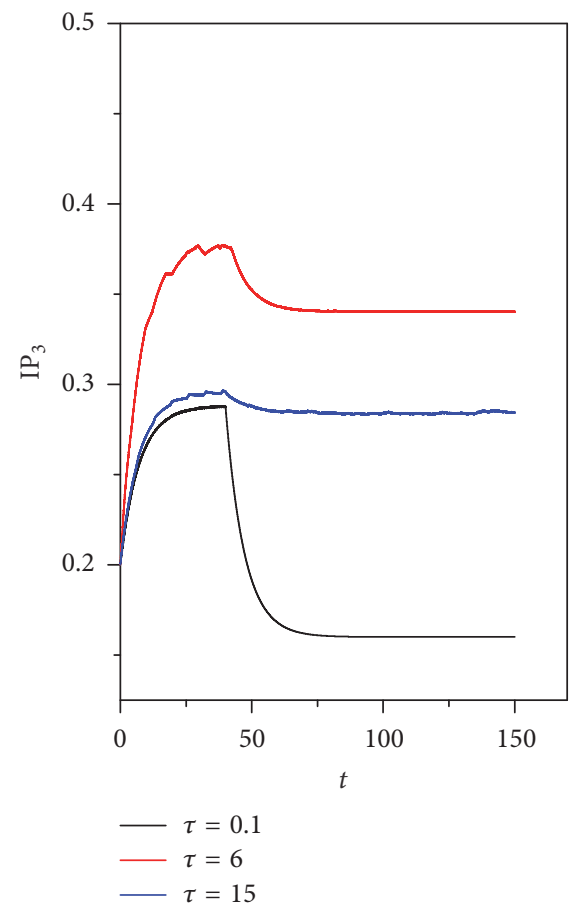

(b)

FIGURE 12: Fluctuation in $\mathrm{Ca}^{2+}$ concentration (a) and $\mathrm{IP}_{3}$ concentration (b) is calculated by applying different time delays in autapse with feedback gain $g_{e}=-0.5$. The parameter is set as $r_{\mathrm{IP}_{3}}=0.2$. And the external forcing current regulates the neuron by setting $I_{\text {ext }}=$ $10.0 \mu \mathrm{mol} / \mathrm{L} \cdot \mathrm{cm}^{2}$ at $t<40 \mathrm{~s}$. 


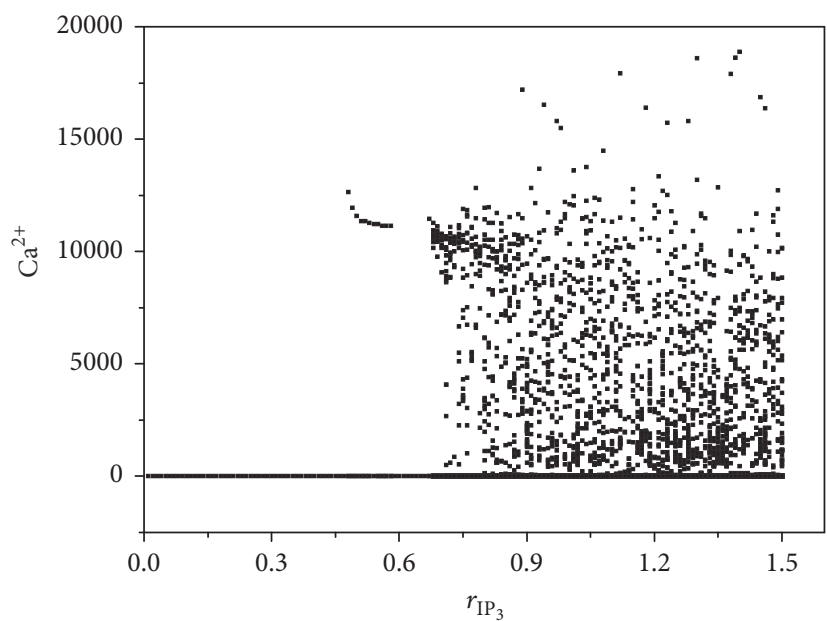

(a)

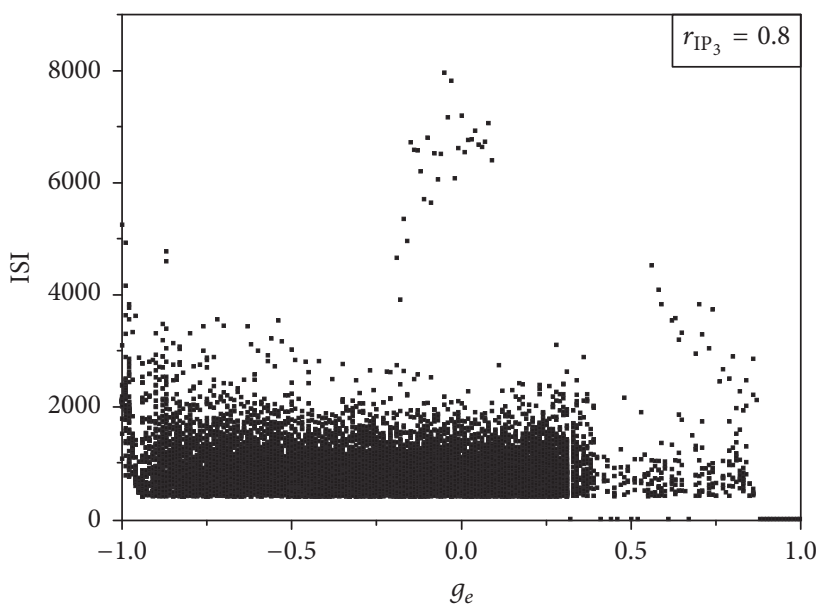

(c)

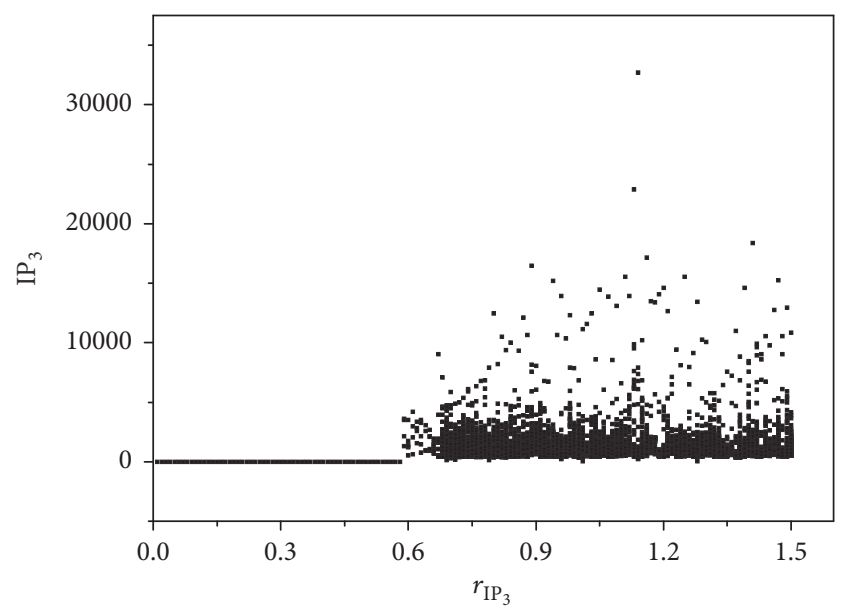

(b)

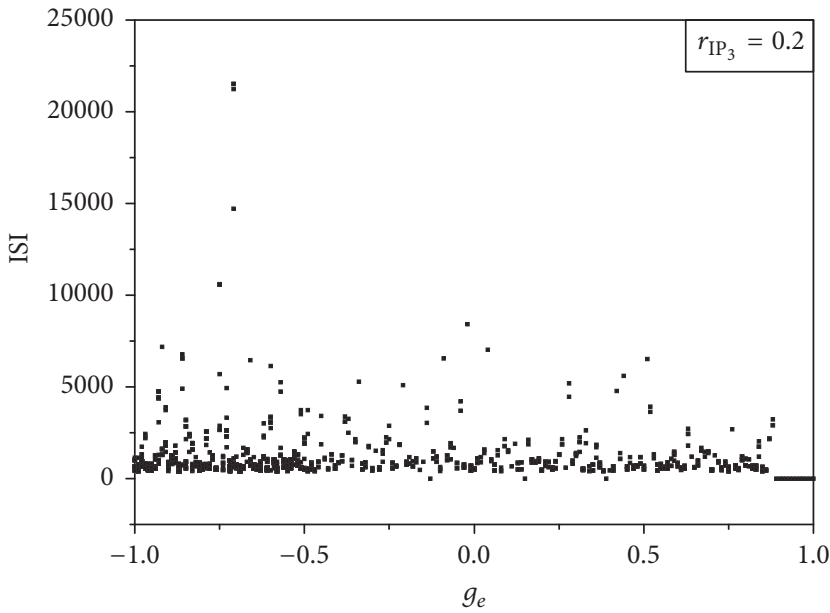

(d)

FIGURE 13: Bifurcation diagram for $\mathrm{Ca}^{2+}, \mathrm{IP}_{3}$, and ISI is calculated by setting different $r_{\mathrm{IP}_{3}}$ and $g_{e}$, respectively.

\section{Conclusions}

Based on the Hodgkin-Huxley neuron model, the effects of astrocyte modulation and autapse connection are considered to set an improved neuron-astrocyte-autapse network model so that the biological function of autapse can be discussed. For the isolate neuron models, the dynamical properties in electrical activities have been extensively investigated to be consistent with the experimental series. The biological role of astrocyte is confirmed on the mode transition of electrical activities in neuron coupled by astrocyte, and action potential is controlled by $\mathrm{Ca}^{2+}$ oscillation and changes of $\mathrm{IP}_{3}$ via release of neurotransmitter. Autapse connection to neuron has confirmed that autaptic modulation could be helpful to enhance excitability of neuron under positive feedback while spiking and bursting behaviors can be suppressed by negative feedback in autapse. As a result, it is important to set a complete neuron model so that the biological function of astrocyte and autapse connection can be estimated completely. Based on our proposed new neuron model, it is found that autapse connection can also be helpful to change the oscillating behaviors for $\mathrm{Ca}^{2+}$ and also the changes of $\mathrm{IP}_{3}$; as a result, electric response to external forcing and mode selection in neuron can be self-adaptive.

The main contribution of this submission could be that we proposed a new neuronal network model developed from the biological $\mathrm{HH}$ model with the biological effect of astrocyte and autapse connection being considered. It explains the biological function of autapse connection and intrinsic exchange of signal in neuron from molecular level. It could be helpful to investigate the collective behavior of neuronal network composed of large number neurons.

\section{Conflicts of Interest}

The authors declare that there are no conflicts of interest regarding the publication of this paper. 


\section{Acknowledgments}

This work is partially supported by the National Natural Science of Foundation of China under the Grant nos. 11672122, 11365014 , and 11372122 .

\section{References}

[1] E. A. Newman, "New roles for astrocytes: regulation of synaptic transmission," Trends in Neurosci, vol. 26, pp. 536-542, 2003.

[2] R. D. Fields and B. Stevens-Graham, "Neuroscience: new insights into neuron-glia communication," Science, vol. 298, no. 5593, pp. 556-562, 2002.

[3] G. Bonvento, C. Giaume, and J. Lorenceau, "Neuron-glia interactions: from physiology to behavior," Journal of Physiology Paris, vol. 96, no. 3, pp. 167-168, 2002.

[4] V. Parpura and P. G. Haydon, "Physiological astrocytic calcium levels stimulate glutamate release to modulate adjacent neurons," Proceedings of the National Academy of Sciences of the United States of America, vol. 97, no. 15, pp. 8629-8634, 2000.

[5] S. J. Smith, "Neural signalling: neuromodulatory astrocytes," Current Biology, vol. 4, no. 9, pp. 807-810, 1994.

[6] T. Fellin, "Communication between neurons and astrocytes: relevance to the modulation of synaptic and network activity," Journal of Neurochemistry, vol. 108, no. 3, pp. 533-544, 2009.

[7] A. C. Charles, J. E. Merrill, E. R. Dirksen, and M. J. Sanderson, "Intercellular signaling in glial cells: calcium waves and oscillations in response to mechanical stimulation and glutamate," Neuron, vol. 6, no. 6, pp. 983-992, 1991.

[8] A. H. Cornell-Bell, S. M. Finkbeiner, M. S. Cooper, and S. J. Smith, "Glutamate induces calcium waves in cultured astrocytes: long-range glial signaling," Science, vol. 247, no. 4941, pp. 470-473, 1990.

[9] J. W. Dani, A. Chernjavsky, and S. J. Smith, "Neuronal activity triggers calcium waves in hippocampal astrocyte networks," Neuron, vol. 8, no. 3, pp. 429-440, 1992.

[10] H. Van Der Loos and E. M. Glaser, "Autapses in neocortex cerebri: synapses between a pyramidal cell's axon and its own dendrites," Brain Research, vol. 48, pp. 355-360, 1972.

[11] A. B. Karabelas and D. P. Purrura, "Evidence for autapses in the substantia nigra," Brain Research, vol. 200, no. 2, pp. 467-473, 1980.

[12] J. M. Bekkers and C. F. Stevens, "Excitatory and inhibitory autaptic currents in isolated hippocampal neurons maintained in cell culture," Proceedings of the National Academy of Sciences of the United States of America, vol. 88, no. 17, pp. 7834-7838, 1991.

[13] J. M. Bekkers, "Synaptic transmission: functional autapses in the cortex," Current Biology, vol. 13, no. 11, pp. R433-R435, 2003.

[14] C. S. Herrmann and A. Klaus, "Autapse turns neuron into oscillator," International Journal of Bifurcation and Chaos in Applied Sciences and Engineering, vol. 14, no. 2, pp. 623-633, 2004.

[15] R. Saada, N. Miller, I. Hurwitz, and A. J. Susswein, "Autaptic excitation elicits persistent activity and a plateau potential in a neuron of known behavioral function," Current Biology, vol. 19, no. 6, pp. 479-484, 2009.

[16] K. Suga, "Isoproterenol facilitates GABAergic autapses in fastspiking cells of rat insular cortex," Journal of Oral Science, vol. 56, no. 1, pp. 41-47, 2014.
[17] H. Wang, L. Wang, Y. Chen, and Y. Chen, "Effect of autaptic activity on the response of a Hodgkin-Huxley neuron," Chaos. An Interdisciplinary Journal of Nonlinear Science, vol. 24, no. 3, Article ID 033122, 13 pages, 2014.

[18] X. L. Song, C. N. Wang, J. Ma, and J. Tang, "Transition of electric activity of neurons induced by chemical and electric autapses," Science China Technological Sciences, vol. 58, no. 6, pp. 10071014, 2015.

[19] D. Guo, M. Chen, M. Perc et al., "Firing regulation of fastspiking interneurons by autaptic inhibition," EPL, vol. 114, no. 3, Article ID 30001, 2016.

[20] J. Ma, X. Song, J. Tang, and C. Wang, "Wave emitting and propagation induced by autapse in a forward feedback neuronal network," Neurocomputing, vol. 167, pp. 378-389, 2015.

[21] J. Ma, H. Qin, X. Song, and R. Chu, "Pattern selection in neuronal network driven by electric autapses with diversity in time delays," International Journal of Modern Physics B, vol. 29, no. 1, Article ID 1450239, 2015.

[22] E. Yilmaz, V. Baysal, M. Ozer, and M. z. Perc, "Autaptic pacemaker mediated propagation of weak rhythmic activity across small-world neuronal networks," Physica A. Statistical Mechanics and its Applications, vol. 444, pp. 538-546, 2016.

[23] J. Ma and J. Tang, "A review for dynamics of collective behaviors of network of neurons," Science China Technological Sciences, vol. 58, no. 12, pp. 2038-2045, 2015.

[24] C. Wang, S. Guo, Y. Xu et al., "Formation of autapse connected to neuron and its biological function," Complexity, vol. 2017, Article ID 5436737, 9 pages, 2017.

[25] F. Wu, C. Wang, Y. Xu et al., "Model of electrical activity in cardiac tissue under electromagnetic induction," Scientific Reports, vol. 6, Article ID 28, 2016.

[26] M. Lv and J. Ma, "Multiple modes of electrical activities in a new neuron model under electromagnetic radiation," Neurocomputing, vol. 205, pp. 375-381, 2016.

[27] M. Lv, C. Wang, G. Ren, J. Ma, and X. Song, "Model of electrical activity in a neuron under magnetic flow effect," Nonlinear Dynamics, vol. 85, no. 3, pp. 1479-1490, 2016.

[28] Y. Wang, J. Ma, Y. Xu, F. Wu, and P. Zhou, “The electrical activity of neurons subject to electromagnetic induction and Gaussian white noise," International Journal of Bifurcation and Chaos, vol. 27, no. 2, Article ID 1750030, 12 pages, 2017.

[29] Y. Xu, H. Ying, Y. Jia et al., "Autaptic regulation of electrical activities in neuron under electromagnetic induction," Scientific Reports, vol. 7, Article ID 43452, 2017.

[30] S. Nadkarni and P. Jung, "Spontaneous oscillations of dressed neurons: a new mechanism for epilepsy?" Physical Review Letters, vol. 91, no. 26, Article ID 268101, 2003.

[31] D. E. Postnov, L. S. Ryazanova, N. A. Brazhe et al., "Giant glial cell: new insight through mechanism-based modeling," Journal of Biological Physics, vol. 34, no. 3, pp. 441-457, 2008.

[32] P. Allegrini, L. Fronzoni, and D. Pirino, "The influence of the astrocyte field on neuronal dynamics and synchronization," Journal of Biological Physics, vol. 35, no. 4, pp. 413-423, 2009.

[33] M. Amiri, N. Hosseinmardi, F. Bahrami et al., "Astrocyteneuron interaction as a mechanism responsible for generation of neural synchrony: a study based on modeling and experiments," Journal of Computational Neuroscience, vol. 34, no. 3, pp. 489-504, 2013.

[34] J. Tang, J. M. Luo, and J. Ma, "Information transmission in a neuron-astrocyte coupled model," PLoS ONE, vol. 8, no. 11, Article ID e80324, 2013. 
[35] J. Tang, J. Zhang, J. Ma et al., "Astrocyte calcium wave induces seizure-like behavior in neuron network," Science China Technological Sciences, pp. 1-8, 2016.

[36] X. Song, C. Wang, J. Ma, and G. Ren, "Collapse of ordered spatial pattern in neuronal network," Physica A. Statistical Mechanics and its Applications, vol. 451, pp. 95-112, 2016.

[37] J. Ma, Y. Xu, G. Ren, and C. Wang, "Prediction for breakup of spiral wave in a regular neuronal network," Nonlinear Dynamics. An International Journal of Nonlinear Dynamics and Chaos in Engineering Systems, vol. 84, no. 2, pp. 497-509, 2016.

[38] W.-T. Yu, J. Tang, J. Ma et al., "Heterogeneous delay-induced asynchrony and resonance in a small-world neuronal network system," EPL, vol. 114, no. 5, Article ID 50006, 2016.

[39] E. Yilmaz, M. Ozer, V. Baysal, and M. Perc, "Autapse-induced multiple coherence resonance in single neurons and neuronal networks," Scientific Reports, vol. 6, Article ID 30914, 2016.

[40] H. G. Gu, B. B. Pan, and Y. Y. Li, "The dependence of synchronization transition processes of coupled neurons with coexisting spiking and bursting on the control parameter, initial value, and attraction domain," Nonlinear Dynamics, vol. 82, no. 3, pp. 1191-1210, 2015.

[41] R. Uzun, M. Ozer, and M. Perc, "Can scale-freeness offset delayed signal detection in neuronal networks?” EPL, vol. 105, no. 6, Article ID 60002, 2014.

[42] Q. Wang, H. Zhang, M. z. Perc, and G. Chen, "Multiple firing coherence resonances in excitatory and inhibitory coupled neurons," Communications in Nonlinear Science and Numerical Simulation, vol. 17, no. 10, pp. 3979-3988, 2012.

[43] X. Sun, J. Lei, M. Perc et al., "Burst synchronization transitions in a neuronal network of subnetworks," Chaos, vol. 21, no. 1, Article ID 016110, 2011.

[44] Q. Wang, G. Chen, and M. Perc, "Synchronous bursts on scalefree neuronal networks with attractive and repulsive coupling," PLoS ONE, vol. 6, no. 1, Article ID e15851, 2011.

[45] H. Gu and Z. Zhao, "Dynamics of time delay-induced multiple synchronous behaviors in inhibitory coupled neurons," PLoS ONE, vol. 10, no. 9, Article ID e0138593, 2015.

[46] D. Fan, Q. Wang, and M. Perc, "Disinhibition-induced transitions between absence and tonic-clonic epileptic seizures," Scientific Reports, vol. 5, Article ID 12618, 2015.

[47] Y.-X. Li and J. Rinzel, "Equations for $\mathrm{InsP}_{3}$ receptor-mediated $\left[\mathrm{Ca}^{2+}\right]_{i}$ oscillations derived from a detailed kinetic model: a Hodgkin-Huxley like formalism," Journal of Theoretical Biology, vol. 166, no. 4, pp. 461-473, 1994.

[48] P. Bezzi, G. Carmignoto, L. Pasti et al., "Prostaglandins stimulate calcium-dependent glutamate release in astrocytes," Nature, vol. 391, no. 6664, pp. 281-285, 1998.

[49] L. Pasti, A. Volterra, T. Pozzan, and G. Carmignoto, "Intracellular calcium oscillations in astrocytes: a highly plastic, bidirectional form of communication between neurons and astrocytes in situ," The Journal of Neuroscience, vol. 17, no. 20, pp. 7817-7830, 1997.

[50] H. R. Parri, T. M. Gould, and V. Crunelli, "Spontaneous astrocytic $\mathrm{Ca}^{2+}$ oscillations in situ drive NMDAR-mediated neuronal excitation," Nature Neuroscience, vol. 4, no. 8, pp. 803812, 2001.

[51] W.-L. Duan and C. Zeng, "Statistics for anti-synchronization of intracellular calcium dynamics," Applied Mathematics and Computation, vol. 293, pp. 611-616, 2017.

[52] W.-L. Duan and C. Zeng, "Signal power amplification of intracellular calcium dynamics with non-Gaussian noises and time delay," Applied Mathematics and Computation, vol. 292, pp. 400-405, 2017.

[53] W.-L. Duan and C. Zeng, "Role of time delay on intracellular calcium dynamics driven by non-Gaussian noises," Scientific Reports, vol. 6, Article ID 25067, 2016.

[54] C. Zeng, C. Zeng, A. Gong, and L. Nie, "Effect of time delay in FitzHughNagumo neural model with correlations between multiplicative and additive noises," Physica A: Statistical Mechanics and its Applications, vol. 389, no. 22, pp. 5117-5127, 2010.

[55] S. Guo, C. Wang, J. Ma, W. Jin et al., “Transmission of blocked electric pulses in a cable neuron model by using an electric field," Neurocomputing, vol. 215, pp. 627-637, 2016. 


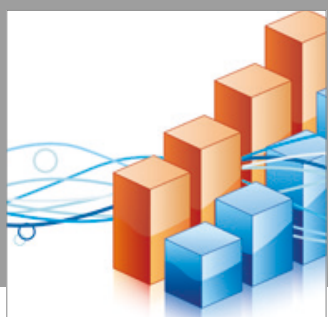

Advances in

Operations Research

vatersals

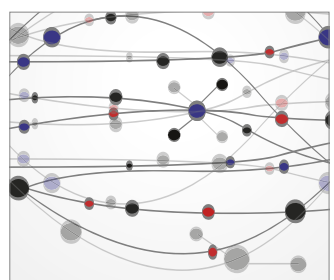

\section{The Scientific} World Journal
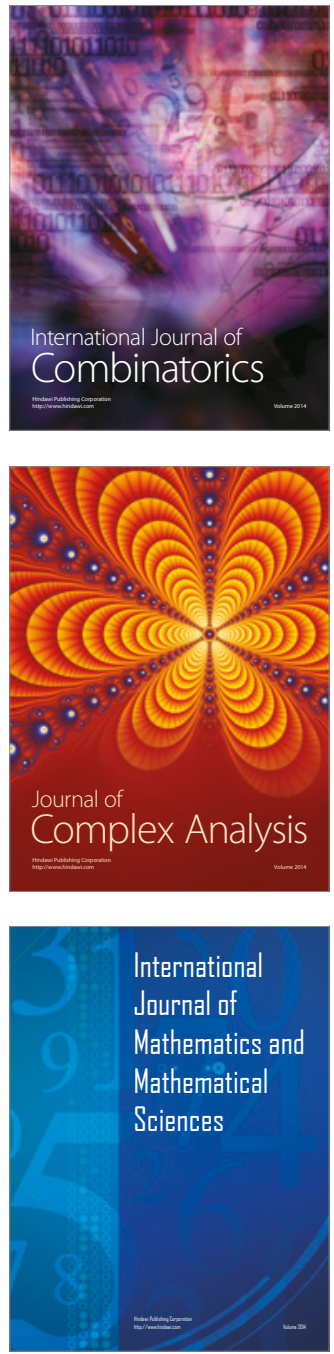
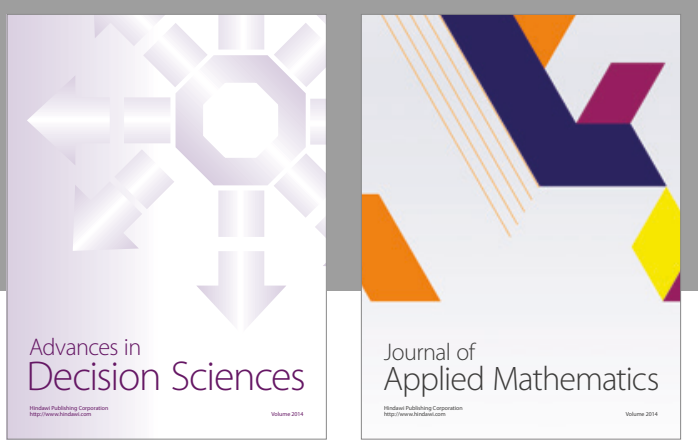

Algebra

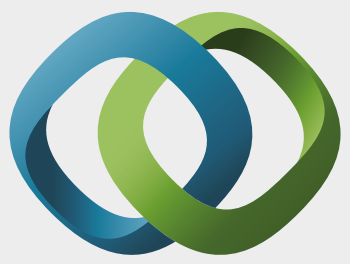

\section{Hindawi}

Submit your manuscripts at

https://www.hindawi.com
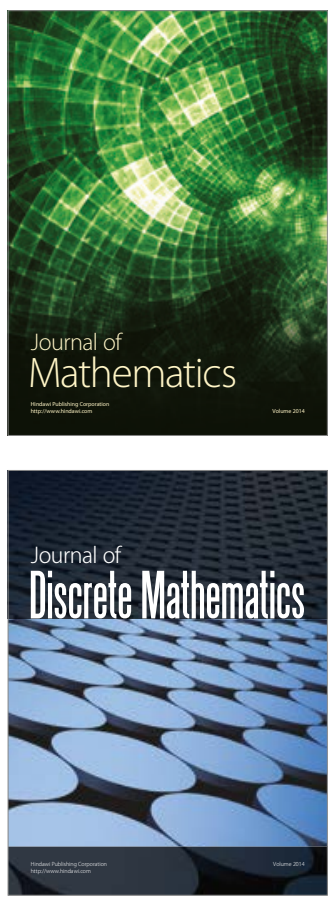

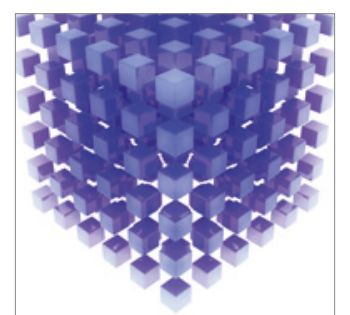

Mathematical Problems in Engineering
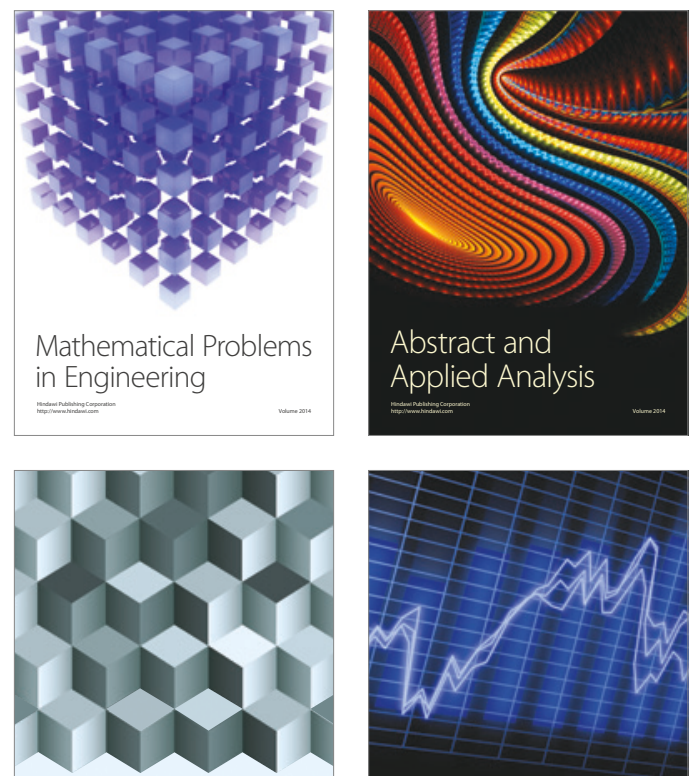

Journal of

Function Spaces

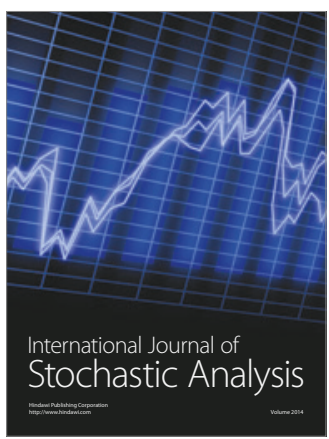

Probability and Statistics
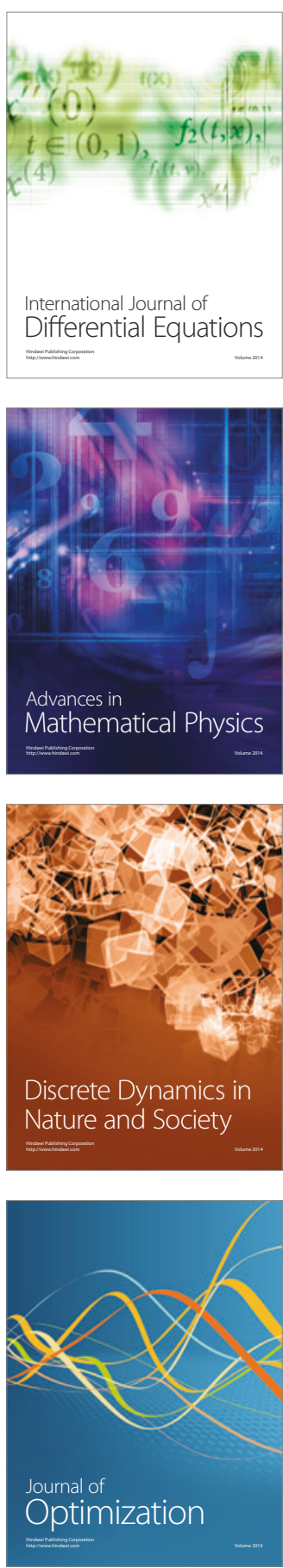